\title{
COVID-19 outbreak and impact on mental health and psychiatric services
}

\author{
Carlo Fraticelli, Alberto Alamia, Ester Guanella \\ Department of Mental Health and Addictions - ASST Lariana, Italy
}

\section{Introduction}

The spread of COVID-19 is having such an impact on the Italian Regional Health System (IRHS) that it had to drastically restructure in a matter of days. All non-essential activities and deferrable operation were suspended, as well as private healthcare activities, in order to make available as much workforce and resources as possible. Hospital wards have been converted in COVID units, and non-critical patients have to be discharged before optimal time in order to make space for the growing need of hospital beds for COVID-19 patients. Emergency Departments (EDs) had to reshape to make dedicated access, areas and routes for COVID-19 patients, in order to minimize the risk of contagion for non-infected subjects. In most EDs a pre-triage has been arranged outside the hospital with adapted field tents.

The major concern was about the high numbers of patients needing life support interventions, which is currently overwhelming the capacity of EDs and Intensive Care Units (ICU).

Healthcare workers went through a further workload that also involved non-emergency departments. They have to face enormous pressure including a high risk of infection and shortage of individual protection devices, ventilators, individual protection equipment, oxygen, disinfectants and essential stuff in general. Human resources themselves are lacking setting one of the biggest problems in the whole situation.

Outbreaks affect individuals and society at many levels and show significant psychological disruptions. Mental health services must be ready to face a potential outbreak of mental illnesses as a consequence of the widespread psychological stressors connected with the epidemiological crisis. Psychiatrists and other mental health workers are in a unique and central condition to support at several level individuals, communities and institutions. ${ }^{2}$

Correspondence: Carlo Fraticelli, Department of Mental Health and Addictions - ASST Lariana, Via Napoleona 60, 22100 Como, Italy Tel.: +39.031.585.5958/9597 - Fax: +39.031.5855438/8640

E-mail: carlo.fraticelli@asst-lariana.it

Key words: COVID-19; outbreak; mental health; health care workers. Conflict of interest: the authors declare no potential conflict of interest.

Received for publication: 16 April 2020.

Accepted for publication: 22 April 2020.

This work is licensed under a Creative Commons Attribution 4.0 License (by-nc 4.0).

(C) Copyright: the Author(s), 2020

Licensee PAGEPress, Italy

Emergency Care Journal 2020; 16:9040

doi:10.4081/ecj.2020.9040

\section{Mental Health in general population}

The epidemic crisis is having a huge impact on the mental health of the general population, even on those people otherwise mentally sane. The threat of infectious diseases can manifest as anxiety and panic. Personal reactions may differ, but the fear of getting sick can be amplified by pre-existing anxiety and depressive conditions. On the other hand, patients with severe mental illness who are concerned about epidemics may risk relapses due to treatment discontinuation, become careless and take on excessive risk. Anxiety and a feeling of helplessness can encourage the adoption of unhealthy behaviors, as the substance abuse. ${ }^{2}$

In literature are widely reported negative psychological effects including post-traumatic stress symptoms, confusion, and anger as a result of extreme social distancing measures like quarantine. ${ }^{3}$ Stress factors included longer quarantine duration, fears of infection, frustration, separation from the beloved, boredom, inadequate supplies and information, financial losses, and stigma. Some researchers have suggested long-lasting effects of quarantine such as vigilant handwashing and avoidance of crowds and, for some, the return to normality was delayed by many months. ${ }^{4}$ Psychological effects can be long-lasting and have a wide range of manifestations. The incidence of mental health sequelae was studied in the aftermath of the Ebola epidemic in West Africa. A study found that $6 \%$ of the survivors and family members were suffering from anxiety-depression 1 year after the epidemic, and 16\% met levels of Post-Traumatic Stress Disorder (PTSD). ${ }^{5}$

Psychiatrists can empower patients, families, and health providers through education about the psychological consequences of social distancing and quarantine. It is essential for designated health officials to provide clear communication, updated information, basic supplies (such as food, water, and medical supplies), and reinforce the sense of altruism.

\section{Mental health in healthcare workers}

Healthcare workers taking care of the most severe cases in ICU and COVID units are continuously experiencing substantial psychological distress. The huge amount of work overload, isolation and fear for personal and relatives safety is exposing them to a high risk of mental health problems. ${ }^{6}$ Frequently reported symptoms among nurses and physicians are depression, anxiety, insomnia, and emotional distress. In a Chinese study nurses, women and frontline workers reported more severe symptoms on all measurements. ${ }^{7}$ Among the socio-demographic features, the common risk factors for stress, depression, and anxiety symptoms are: feminine gender, more than 10 years of working, history of mental disorders, history of concomitant chronic diseases, and family members or relatives confirmed suspected COVID- 19 .

The COVID-19 epidemic is forcing health care professionals to optimize scarce resources to allocate them to equally needy patients. Intense feelings of shame, guilt, or inadequacy contribute to the onset of psychiatric symptoms such as depression, post-traumatic 
stress disorders, and suicidal thoughts. ${ }^{8}$ In the SARS outbreak in 2003 , about $10 \%$ of the healthcare providers had experienced high levels of post-traumatic stress symptoms. ${ }^{9}$ Being quarantined or have worked in high-risk wards during SARS outbreak resulting in higher alcohol use in healthcare workers even after 3 years from exposition. ${ }^{10}$ The NICE (National Institute for Health and Care Excellence) recommends "active monitoring" of healthcare staff under extreme pressure to identify and support suffering people and avoid long lasting damage. ${ }^{11}$ Psychological problems can affect the medical workers' cognitive functioning and decision-making ability. Promoting health workers self-care is essential to support the general health care system. Providing support to colleagues, finding constructive solutions to job challenges, and staying connected with family and friends helps to reduce environmental impact and overall stress. Recognizing personal experiences, expectations and fears of healthcare professionals requires much more needs to optimize safety in their living environment. For front-line clinicians, the worries about transmitting the virus to family members will need to be addressed. While healthcare professionals often accept an increased risk of infection, as part of their professional choice, they often show great concern about the transmission to family members, particularly for elderly one, immunocompromised or suffering from chronic diseases. Psychological interventions and care measures should be integrated with a shift system that allows frontline health workers to rest and alternate in high-pressure roles.

\section{Outbreak and patients with psychiatric disease}

Italian outpatient mental health services had to suspend all non-urgent and routine interventions in line with the social distancing disposition imposed by the government, limiting their focus on compelling psychiatric emergencies only. This set some concern about those outpatients who are not receiving adequate psychiatric care anymore. Mentally ill patients may have difficulty complying with the containment measures due to cognitive impairment, little awareness of risk, and diminished efforts regarding personal protection. Moreover, psychiatric patients infected with COVID-19 can be exposed to greater barriers in obtaining timely health services. $^{12}$ People with psychiatric conditions usually have an increased risk to develop medical comorbidities than the general population such as cardiovascular disease, diabetes mellitus, hypertension, chronic lung disease. ${ }^{13}$ Such conditions have been associated with more severe evolution and higher mortality risk in COVID-19 patients. ${ }^{14}$ In particular, individuals with chronic medical conditions should be educated and encouraged by mental health workers to follow general measures to reduce transmission of infection (hand washing, respiratory hygiene, avoiding touching the face, avoiding crowds). Personal history of psychiatric patients could expose them to be more emotionally sensitive to the COVID-19 epidemic, with relapses or worsening of an existing mental disease due to the high susceptibility to stress compared to the general population. ${ }^{15}$ In addition, the reduction in outpatient activities due to the regulations imposed by regional and national authorities resulted in a significant interference with the continuity of care and a reduction in contacts.

\section{Conclusions}

Psychiatric services could play a key role in supporting frontline healthcare workers, helping them to carry the burden of work overload, stressing working conditions, exposure to several trau- matic events and increased risk of getting sick. At the same time, mental health facilities have to provide psychosocial support to affected individuals (e.g., during the acute stage of the illness, during laboratory testing periods, and before discharge) and their relatives. These objectives could also be achieved through strengthening family and community networks services.

In addition, today mental health providers have to consider what will come next. It is foreseeable that after the infection outbreak starts to subside, at a moment of profound pain from loss of a loved one, uncertainty, experience of survivor's guilt, resumption (and fear) of social contacts, and financial hardships a wide variety of mental problems will occur in affected people, their families, and the whole community.

\section{References}

1. Coen D, Paolillo C, Cavazza M, et al. Changing emergency department and hospital organization in response to a changing epidemic. Emerg Care J 2020,16:8969.

2. Huremović D (ed). Psychiatry of Pandemics: A Mental Health Response to Infection Outbreak. Cham, Switzerland: Springer Nature Switzerland AG;2019.

3. Brooks SK, Webster RK, Smith LE, et al. the psychological impact of quarantine and how to reduce it: rapid review of the evidence. Lancet 2020;395:912-20.

4. Cava MA, Fay KE, Beanlands HJ, et al. The experience of quarantine for individuals affected by SARS in Toronto. Public Health Nurs 2005;22:398-406.

5. Jalloh MF, Li W, Bunnell RE, et al. Impact of Ebola experiences and risk perceptions on mental health in Sierra Leone, July 2015. BMJ Glob Health 2018;3:e000471.

6. Maunder RG. Was SARS a mental health catastrophe? Gen Hosp Psychiatry 2009;31:316-7.

7. Jianbo L, Simeng M, Ying W, et al. Factors associated with mental health outcomes among health care workers exposed to coronavirus disease 2019. JAMA Network Open. 2020;3(3): e203976.

8. Greenberg N, Docherty M, Gnanapragasam S, Wessely S. Managing mental health challenges faced by healthcare workers during covid-19 pandemic. BMJ 2020;368: $\mathrm{m} 1211$

9. Wu P, Fang Y, Guan Z, et al. The psychological impact of the SARS epidemic on hospital employees in China: exposure, risk perception, and altruistic acceptance of risk. Can J Psychiatr 2009;54:302-11.

10. Wu P, Liu X, Fang Y, et al. Alcohol abuse/dependence symptoms among hospital employees exposed to a SARS outbreak. Alcohol Alcohol 2008;43:706-12.

11. National Institute for Health and Care Excellence. Post-traumatic stress disorder. Available from: NG116.2018. https://www.nice.org.uk/guidance/ng116

12. Sartorius N. Comorbidity of mental and physical disorders: a key problem for medicine in the 21st century. Acta Psychiatrica Scandinavica 2018;137:369-70.

13. Kivimäki M, Batty GD, Pentti J, et al. Association between socioeconomic status and the development of mental and physical health conditions in adulthood: a multi-cohort study. Lancet Public Health 2020;5:30248-8.

14. Zhou F, Yu T, Du R, et al. Clinical course and risk factors for mortality of adult inpatients with COVID-19 in Wuhan, Chian: a retrospective cohort study. Lancet 2020;395:1054-62.

15. Yao H, Chen JH, Xu YF. Patients with mental health disorders in the COVID-19 epidemic. Lancet Psychiatry 2020;7:e21 